\title{
Development of Ocular Surface Tribometer and Frictional Characteristics of Human Ocular Surface
}

\author{
Sarwo Pranoto ${ }^{1} 4^{*}$, Shingo Okamoto ${ }^{1}$, Ryoichiro Kataoka1 ${ }^{1}$, Jae Hoon Lee ${ }^{1}$, Atsushi Shiraishi², \\ Yuri Sakane ${ }^{2}$, Masahiko Yamaguchi ${ }^{3}$, Yuichi Ohashi ${ }^{2}$ \\ ${ }^{1}$ Ehime University, Graduate School of Science and Engineering, Mechanical Engineering Course, \\ Matsuyama, Japan. \\ 2 Ehime University School of Medicine, Department of Ophthalmology, Toon, Japan. \\ ${ }^{3}$ Ehime Prefectural Central Hospital, Department of Ophthalmology, Kasuga-tyo 83, Matsuyama, Japan. \\ ${ }^{4}$ Politeknik Negeri Ujung Pandang, Department of Electrical Engineering, Makassar, Indonesia.
}

* Corresponding author. Tel.: +818029952830; email: c861004y@mails.cc.ehime-u.ac.jp Manuscript submitted November 15, 2017; accepted January 15, 2018.

doi: 10.17706/ijbbb.2018.8.2.89-99

\begin{abstract}
The purpose of this research is to determine the frictional characteristics of human ocular surfaces. An ocular surface tribometer was developed to measure the normal forces, frictional forces, and velocities of the probe on human ocular surfaces simultaneously. In this research, the measurement on six trial subjects was performed with the developed ocular surface tribometer. In addition, a mathematical model was proposed to describe the frictional coefficient of the human ocular surface. The frictional characteristic curves were found by using the computational program employing BSG-Starcraft of PSO and LSM developed by the authors in the previous research. It was possible to classify the frictional characteristics of human ocular surfaces into three types.
\end{abstract}

Key words: Dry eye, frictional characteristic, ocular surface tribometer, particle swarm optimization.

\section{Introduction}

In recent years, various researches on a dry eye syndrome have increased. The researches have shown that a dry eye syndrome is mainly caused by a deficiency of tear fluid and an excessive evaporation of the tear [1]. In dry eye patients, the tear fluid is deficient to separate the eyelid and ocular surfaces [2]. Moreover, the eyelid surface where the tear fluid is deficient could be harmed due to the friction between the eyelid and ocular surfaces during blinking [3]. In addition, the eyelid pressure during blinking may change the shape of cornea [4]. Thus, various instruments have been developed by researchers to measure the eyelid pressure on the ocular surface [5]-[7]. One of the instruments, the blepharo-tensiometer that uses a tactile pressure sensor for measuring eyelid pressure was developed by the authors [8].

In addition, in the previous research by the authors, the ocular surface tribometer was developed for measuring frictional coefficients of human ocular surfaces [9]. Then the computational program employing the genetic algorithm and the LSM for determining frictional characteristics of human ocular surfaces was developed. The ocular surface tribometer consists of the frictional coefficient measuring apparatus and the device to measure the moving velocity of the probe. However, the ocular surface tribometer has low accuracy in the measurement of frictional coefficients of human ocular surfaces because a strain gauge was used as the two-axis force sensor (TL701 Handy Rub Tester, Trinity Lab, Japan). Moreover, the data on 
normal forces, frictional forces, and frictional coefficients measured by the previous ocular surface tribometer could not be synchronized completely with the data on rotational angle by the encoder since the apparatus for measuring normal forces, frictional forces, and frictional coefficients and the device for measuring the moving velocity of the probe work independently.

Besides, there is an increase on application of optimization algorithms such as PSO (Particle Swarm Optimization) to solve optimization problems. Among many variants of PSO, the BSG (BattleStar Galatica)-Starcraft of PSO has been developed and evaluated to solve some optimization test functions [10]. In the previous research by the authors, the computational program employing BSG-Starcraft of PSO and LSM was developed to determine frictional characteristics of human ocular surface [11].

In the present research, the new ocular surface tribometer was developed in order to solve the problems of the previous ocular surface tribometer. The new ocular surface tribometer that uses an electrostatic capasitive sensor as a two-axis force sensor (Tech Alpha, Japan) was developed in order to increase the accuracy in measuring frictional coefficients. In addition, a new data logger also was developed. By using the new data logger, the data on normal forces, frictional forces, frictional coefficients, and data on rotational angle by the encoder were sampled synchronously. Then, the new ocular surface tribometer was used to measure the normal forces, frictional forces, and velocities of the probe on human ocular surfaces of test subjects simultaneously. Then the frictional characteristic curves of human ocular surfaces were calculated by using the computational program employing the BSG-Starcraft of PSO and the LSM developed by the authors in the previous research.

\section{Frictional Coefficients of Human Ocular Surface}

\subsection{Development of Ocular Surface Tribometer for Measuring the Frictional Coefficients}

In the field of mechanical engineering, to identify the frictional coefficients on journal bearings, the Hersey Number is commonly applied. The Hersey Number [12] is shown as in (1).

$$
H_{s}=\eta \omega / p
$$

where $\eta$ is the viscosity of lubricating oil, $\omega$ the rotational speed of a shaft, and $p$ the pressure of lubricating oil behind the location of the minimum separation between the bearing and the shaft.

In this research, the frictional coefficient, $\mu$ of a human ocular surface is considered to be related to the viscosity, $\eta$ of tear fluid, the velocity, $V_{n}$ of nictation, and the palpebral pressure, $P$. Therefore, a new ocular surface tribometer capable of measuring the moving velocity, $V$ of the probe, the normal force, $N$, and the frictional force, $F$ was developed.

Fig. 1 shows the moving directions of the probe in the ocular surface tribometer on the left eye. The probe is contacted on the human ocular surface by moving it in the $Z$ direction. Then the probe is moved in the $X$ direction to measure $N, F$, and $V$ of the probe.

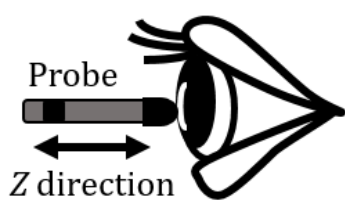

(a) Left side view

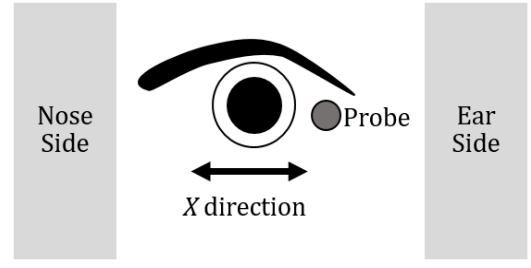

(b) Front view

Fig. 1. Moving directions of the probe in ocular surface tribometer on the left eye. 
Fig. 2 shows the ocular surface tribometer consisting the frictional coefficient measuring apparatus and the device to measure the moving velocity of the probe. An electrostatic capacity type force sensor (Tech Alpha, Japan) was used as the two-axis force sensor (8). The frictional coefficient measuring apparatus was used to measure $N$ and $F$ acquired by the probe. The probe (15is made of stainless steel and has a spherical ball of $3 \mathrm{~mm}$ diameter on its tip. The device to measure $V$ of the probe consists of a frame to fix a face (1), an encoder (4), two pulleys (3), a belt (11) a camera (9), and Light Emitting Diodes (LEDs) (10).

A new data logger which consists of hardware and software was developed. The hardware consists of a signal conditioner and a signal processor. The signal conditioner was used to change the voltages of the normal forces and the frictional forces. The voltages were adjusted to the input voltage range of the signal processor. The signal processor includes a micro-controller (Arduino Due) to convert the analog data of normal forces and frictional forces to the digital ones. In addition, the micro-controller was used to transfer the data on $N, F$, and rotational angle, $\theta_{p}$ of the pulleys measured with the encoder to a laptop-computer via serial communication. The laptop-computer was used to operate the data logger software developed in this research. The software in data logger was used to convert the angular velocity, $\omega_{p}$ of the pulleys measured with the encoder to $V$ of the probe connected to the belt to rotate the pulleys.

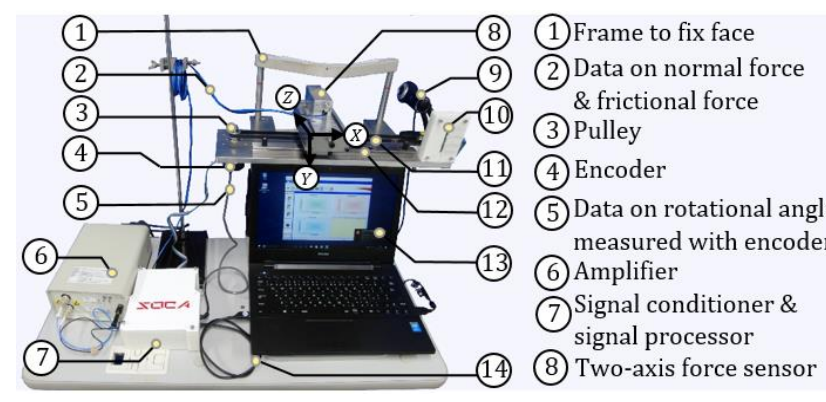

(a) Total device

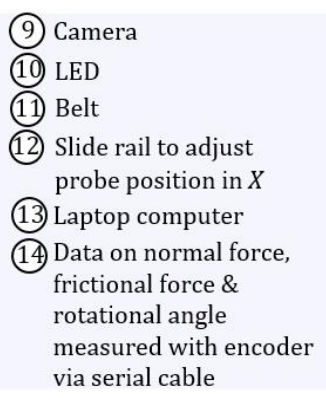

via serial cable

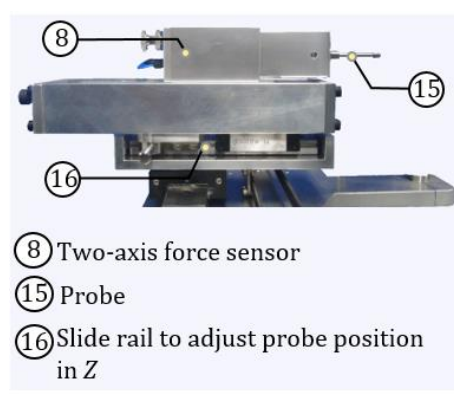

(b) Two-axis force sensor system

Fig. 2. Ocular surface tribometer consisting the frictional coefficient measuring apparatus and the device to measure the moving velocity of the probe.

\subsection{Mathematical Model for Frictional Coefficients}

A tear layer exists between the eyelid and the ocular surface in a normal eye. However, some areas of the eyelid and the ocular surface directly contact each other in a dry eye. Thus, when the eyelid and the ocular surface are fully separated by the tear layer, $\mu$ on a human ocular surface is considered to be within the range of fluid lubrication. While in the condition of the ocular surface is dry, $\mu$ on a human ocular surface is considered to be within the range of mixed lubrication.

In this research, a new number, $X$ was proposed to calculate $\mu$ on the human ocular surface as given in (2).

$$
X=\eta^{p_{1}} V^{p_{2}} / N^{p_{3}}
$$

where parameters, $p_{1}, p_{2}$, and $p_{3}$ are arbitrary real numbers. Then by incorporating the proposed number, $X$, a mathematical model was proposed to describe $\mu$ of the human ocular surface as given in (3).

$$
\mu=p_{4} X^{n-4}+p_{5} X^{n-5}+\ldots+p_{n-1} X+p_{n}
$$

where parameters, $p_{4}, p_{5}, \ldots$, and $p_{n}$ are arbitrary real numbers. In this paper, it is assumed that $\eta$ is constant 
and equal to 1 , in other words $p_{1}=0$.

\section{BSG-Starcraft of PSO}

\subsection{Parameters of Frictional Characteristic Curve on Human Ocular Surface}

In this research, an optimization method was used in determining parameters, $p_{1}, \ldots, p_{n}$ in (2) and (3). Then, a computational program combining the BSG-Starcraft of PSO and the LSM developed by the authors in the previous research [11] was applied to achieve the optimization. The computational program was applied through six steps.

In the first step, the positions, velocities, and inertia weights of all particles in the swarm were initialized. The position and velocity of particle $i$ at iteration $j$ in the $n$-dimensional search space were represented as $x_{i}^{j}=\left(x_{i, 1}^{j}, x_{i, 2}^{j}, \ldots, x_{i, n}^{j}\right)=\left(p_{1}, p_{2}, \ldots, p_{n}\right)$ and $v_{i}^{j}=\left(v_{i, 1}^{j}, v_{i, 2}^{j}, \ldots, v_{i, n}^{j}\right)$, respectively. The initial positions, $x_{i}^{o}$ and velocities, $v_{i}^{0}$ of all particles were randomly generated within pre-defined ranges as expressed in (4) and (5).

$$
\begin{aligned}
& x_{i}^{0}=x_{\text {min }}+\operatorname{rand}\left(x_{\text {max }}-x_{\text {min }}\right) \\
& v_{i}^{O}=v_{\text {min }}+\operatorname{rand}\left(v_{\text {max }}-v_{\text {min }}\right)
\end{aligned}
$$

Here $x_{\min }$ and $x_{\max }$ are the lower and upper bounds on $x$. While $v_{\min }$ and $v_{\max }$ are the lower and upper bounds on $v$. The inertia weight, $w^{j}$ was calculated as in (6).

$$
W^{j}=w_{\max }-\left(\frac{w_{\max }-w_{\min }}{j_{\max }}\right) j
$$

where $w_{\min }$ and $w_{\max }$ are the minimum and maximum inertia weights. In the second step, the objective function was evaluated. The objective function, $F_{i}^{j}$ of particle $i$ at iteration $j$ was given as in (7).

$$
F_{i}^{j}=\sum_{l=1}^{n_{e}} \sqrt{\left(\mu_{i}-\mu_{l}\right)^{2}} \quad\left(i=1 \sim n_{S}\right)
$$

Here $\mu_{l}$ is the actual experimental value of frictional coefficient on the human ocular surface, $n_{e}$ the number of experimental values, and $n_{s}$ the number of particles in a swarm. In this research, the BSG-Starcraft of PSO algorithm was applied to minimize the value of the objective function, $F_{i}^{j}$.

In the third step, the personal best position of particle $i, P_{B e s t, i}$ and the best global position in the current swarm, $G_{B e s t}$ were determined. The $P_{B e s t, i}$ was the smallest value of the objective function, $F_{i}^{j}$ obtained by the particle $i$ at all previous iterations. The smallest value of the objective function among $P_{B e s t, i}$ was determined as $G_{\text {Best }}$

In the fourth step, the $G_{B e s t}$ was determined as the carrier, $X_{\text {Carrier }}$. The $X_{\text {Carrier }}$ was used in each iteration to send some new particles called raptors with the probability 0.9 . The position of raptor $k$ at iteration $j$ in the $n$-dimensional search space was represented as $x_{\text {Raptor } k}^{j}=\left(x_{\text {Raptor } k, 1}^{j}, x_{\text {Raptor } k, 2}^{j}, \ldots, x_{\text {Raptor } k, n}^{j}\right)$. The objective function, $F_{\text {Raptor } k}^{j}$ of raptor $k$ at iteration $j$ was evaluated by using the formula as given in (8).

$$
F_{\text {Raptor k }}^{j}=\sum_{l=1}^{n_{e}} \sqrt{\left(\mu_{k}-\mu_{l}\right)^{2}} \quad\left(k=1 \sim n_{r}\right)
$$


Here $n_{r}$ are the number of raptors in each iteration.

Fig. 3 shows the schematics of raptors exploring the space. If a raptor with better value of objective function than $x_{\text {Carrier }}$ reaches the better position, a jump vector, namely Jump is defined. Then, the swarm jumps to the new position by the translation of the vector Jump. Thus, the new position of $x_{C a r r i e r}$ is now the raptor with the best position. Therefore, due to the new position of the swarm, the $P_{\text {Best, } i}$ and $G_{B e s t}$ are updated.

In the fifth and sixth steps, the velocity and position of particle $i$ were updated as in (9) and (10), respectively.

$$
\begin{gathered}
v_{i}^{j+1}=w^{j} v_{i}^{j}+c_{1} r_{1}\left(P_{\text {Best }, i}-x_{i}^{j}\right)+c_{2} r_{2}\left(G_{\text {Best }}-X_{i}^{j}\right) \\
x_{i}^{j+1}=x_{i}^{j}+v_{i}^{j+1}
\end{gathered}
$$

Here $c_{1}$ is the self-confidence factor and $c_{2}$ the swarm confidence factor. The $r_{1}$ and $r_{2}$ were the random numbers uniformly distributed in the range $(0,1)$.

Fig. 4 shows the updating velocity and position of a particle. As expressed in (9), the velocity, $v_{i}^{j}$ of the particle $i$ is updated by combining $G_{B e s t}$ and $P_{B e s t, i}$ with $w^{j}, c_{1}, c_{2}, r_{1}$, and $r_{2}$. The position, $x_{i}^{j+1}$ of the particle $i$ in the next iteration is affected by the current velocity, $V_{i}^{j}$ of the particle $i, G_{\text {Best }}$ and $P_{\text {Best, } i^{*}}$.

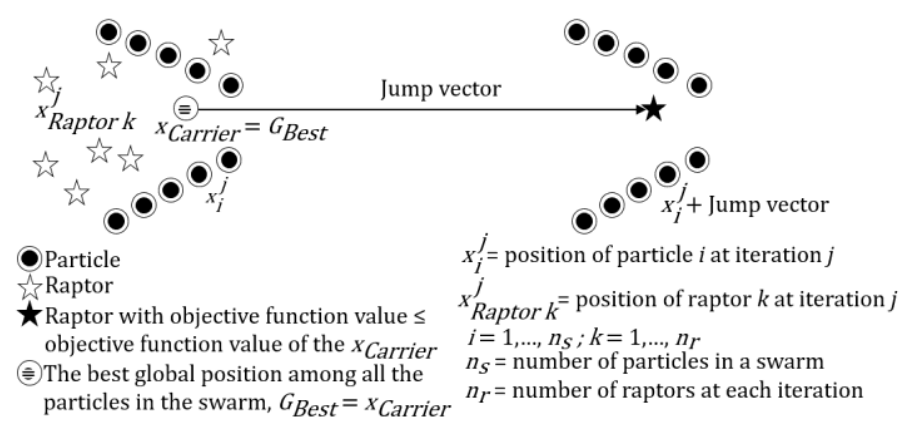

Fig. 3. Schematics of raptors exploring the space.

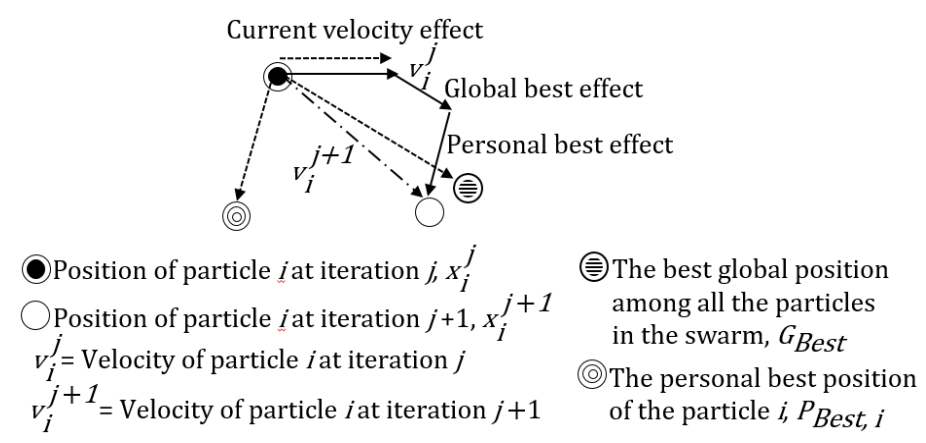

Fig. 4. Updating velocity and position of a particle.

\subsection{Parameter Setting of BSG-Starcraft of PSO}

In this research, the BSG-Starcraft of PSO was employed to determine parameters of frictional characteristic curve on the human ocular surface by minimizing the value of the objective function, $F_{i}^{j}$. Then the number of particles in a swarm, $n_{s}$ was set to 20 . The minimum and the maximum of inertia 
weights, $w_{\min }$ and $w_{\max }$ were set to 0.4 and 0.9 , respectively. While the self-confidence factor, $c_{1}$ and the swarm confidence factor, $c_{2}$ were set equal to 2 . The maximum number of iterations, $j_{\max }$ was set to 200 . In addition, the number of raptors in each iteration, $n_{r}$ was set to 20.

\section{Results}

\subsection{Experimental Data Measured by Ocular Surface Tribometer}

In this research, the measurements were performed on cornea and bulbar conjunctiva of six healthy subjects (subject $i, i=\mathrm{A}, \mathrm{B}, \mathrm{C}, \ldots, \mathrm{F}$ ) by using the developed ocular surface tribometer. For each subject $i$, the measurements on cornea were firstly conducted. The measurements on bulbar conjunctiva were secondly done. In the both measurements, $N, F$, and displacement, $d$ were measured.

In the previous research by the authors, the adoption rate of experimental data measured by the ocular surface tribometer for determining the frictional characteristics of human ocular surfaces was around $80 \%$. In the present research, the 127 data were measured by the new ocular surface tribometer. It was possible to use $96 \%$ of the measurement data for determining the frictional characteristics of human ocular surfaces. The adoption rate of experimental data measured by the new ocular surface tribometer increased because the electrostatic capacitive sensor has the higher accuracy than the strain gauge.

Fig. 5 shows the examples of cornea's data measured by the ocular surface tribometer. Time history responses of $N, F$, and $d$ were measured at the same time using the ocular surface tribometer. In this experiment, $N$ were applied to the cornea within the range of $0.22 \mathrm{gf}$ to $3.10 \mathrm{gf}$. Then, the average values of $N$ and $F$, were calculated from each measured data. The displacements, $d$ of the probe were measured by the encoder and controlled to be within the range of $1.12 \mathrm{~mm}$ to $4.90 \mathrm{~mm}$. Results obtained for the measurements on the bulbar conjunctiva were similar to those of the cornea.

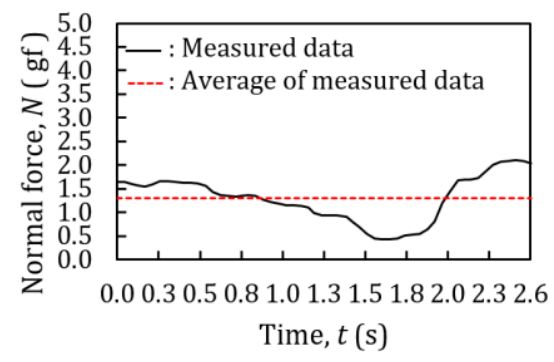

(a) Normal force

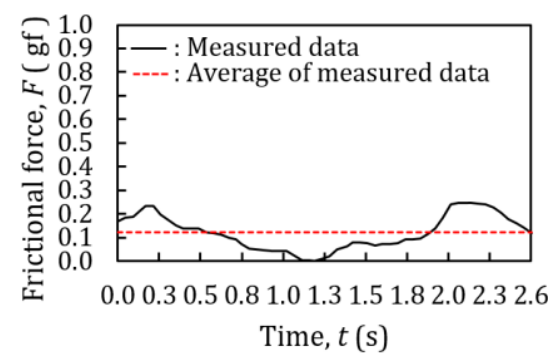

(b) Frictional force

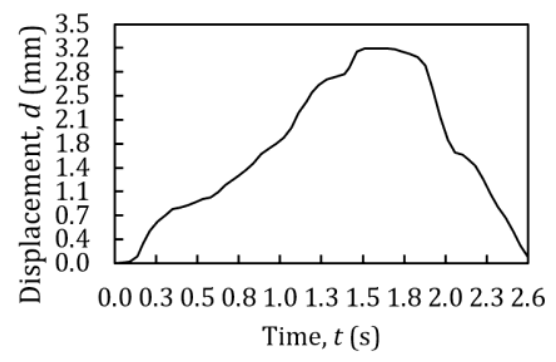

(c) Displacement

Fig. 5. Examples of cornea's data measured by the ocular surface tribometer.

Fig. 6 shows the examples of cornea's results calculated by using the measured data. The measured $N$ and $F$ were used to calculate $\mu$. The measured $d$ of the probe was used to calculate $V$ of the probe. Then, the average values of $\mu$ and $V$ of the probe were calculated. In this experiment, the average values of $\mu$ varied within the range of 0.07 to 0.17 . The average values of $V$ of the probe varied within the range of $1.72 \mathrm{~mm} / \mathrm{s}$ to $3.54 \mathrm{~mm} / \mathrm{s}$. The average values of $\mu$ and $V$ of the probe were used to determine the frictional characteristics of human ocular surface. The calculated results on the bulbar conjunctiva were similar to those of the cornea.

\subsection{Frictional Characteristic Curves of Human Ocular Surface Determined by Using BSG-Starcraft of PSO and LSM}

In this research, the frictional characteristic curves of human ocular surface were calculated by using the BSG-Starcraft of PSO and LSM.

Fig. 7 shows the example of frictional characteristic curve of cornea and bulbar conjunctiva in fluid 
lubrication condition. The frictional characteristic curve is described based on the measured data obtained from the subject $\mathrm{A}$. The parameters, $p_{2}=0.35$ and $p_{3}=0.93$ were obtained when the objection function value, $F_{i}^{j}=0.096$. As for the subject $\mathrm{A}$, the frictional coefficients on both cornea and bulbar conjunctiva fall within the fluid lubrication where the eyelid and ocular surfaces are fully separated by the tear layer. Then the curve in the fluid lubrication shows an upward-sloping characteristic.

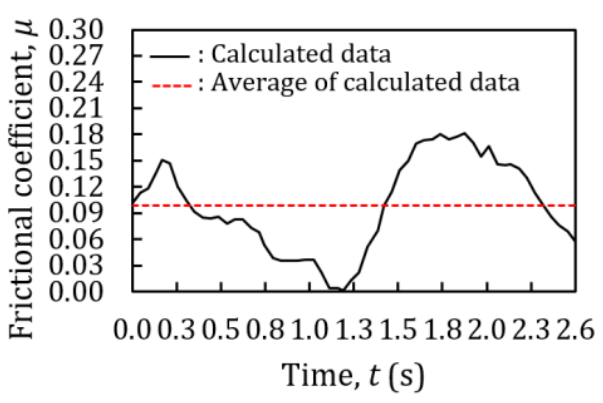

(a) Frictional coefficient

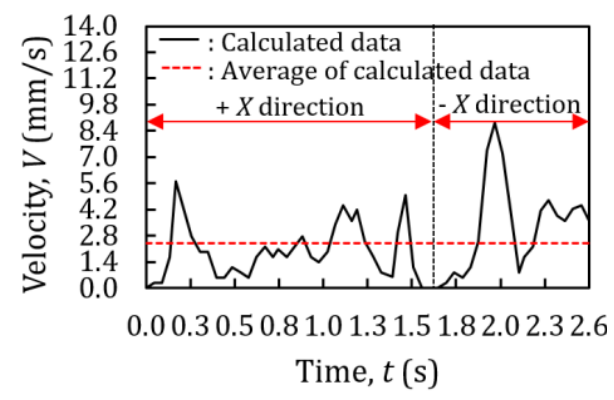

(b) Velocity of probe

Fig. 6. Examples of cornea's results calculated by using the measured data.

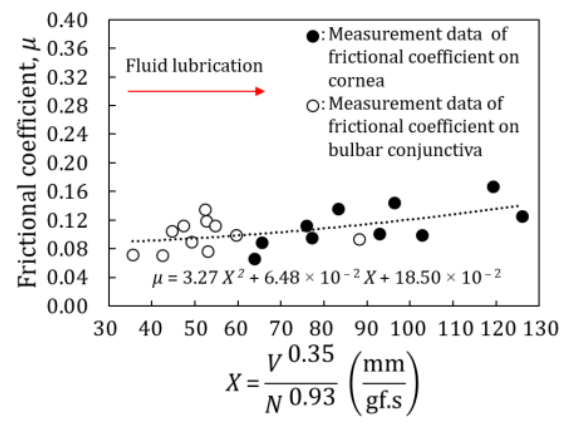

Fig. 7. Example of frictional characteristic curve of cornea and bulbar conjunctiva in fluid lubrication condition.

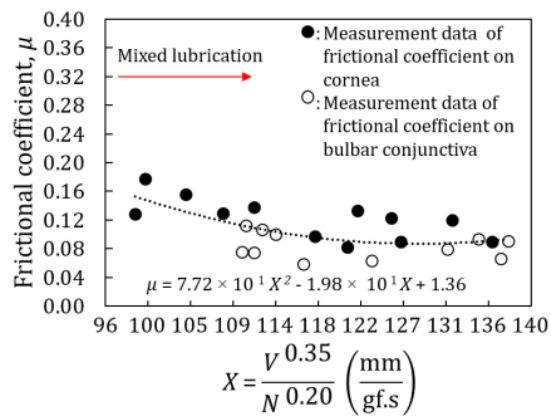

Fig. 8. Example of frictional characteristic curve of cornea and bulbar conjunctiva in mixed lubrication condition.

Fig. 8 shows the example of frictional characteristic curve of cornea and bulbar conjunctiva in mixed lubrication condition. The frictional characteristic curve is described based on the measured data obtained from the subject $\mathrm{B}$. The parameters, $p_{2}=0.35$ and $p_{3}=0.20$ were obtained when the objection function value, $F_{i}^{j}=0.122$. As for the subject $\mathrm{B}$, the frictional coefficients on both cornea and bulbar conjunctiva fall within the mixed lubrication where a part of the eyelid and ocular surfaces is supported by the tear layer, 
and in the other part, the eyelid surface may be in contact with the ocular surface. Then the curve in the mixed lubrication shows a downward-sloping characteristic.

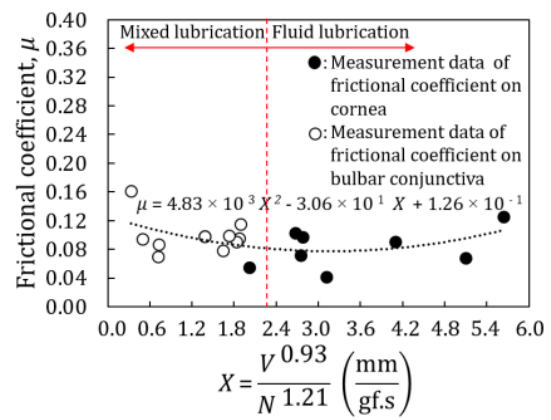

Fig. 9. Example of frictional characteristic curve of cornea and bulbar conjunctiva in lubrication condition containing both mixed and fluid lubrications.

Fig. 9 shows the example of frictional characteristic curve of cornea and bulbar conjunctiva in lubrication condition containing both mixed and fluid lubrications. The frictional characteristic curve is described based on the measured data obtained from the subject $\mathrm{C}$. The parameters, $p_{2}=0.93$ and $p_{3}=1.21$ were obtained when the objection function value, $F_{i}^{j}=0.110$. As for the subject $\mathrm{C}$, the concave upward curve was obtained as the frictional characteristic one. The frictional coefficients on the cornea fall within the fluid lubrication where the eyelid and the cornea are fully separated by the tear layer. Then the curve in the fluid lubrication shows an upward-sloping characteristic. The frictional coefficients on the bulbar conjunctiva fall within the mixed lubrication where a part of the eyelid and bulbar conjunctiva is supported by the tear layer, and in the other part, the eyelid surface may be in contact with the bulbar conjunctiva. Then the curve in the mixed lubrication shows a downward-sloping characteristic.

\section{Conclusion}

The summary of the results is shown below:

(1) The new ocular surface tribometer for measuring frictional coefficients on human ocular surface was developed.

(2) Frictional characteristic curves of the human ocular surfaces have been calculated by using the computational program employing the BSG-Starcraft of PSO and LSM developed by the authors in the previous research [11].

(3) The frictional characteristics of human ocular surfaces could be classified to three types: the fluid lubrication where the eyelid and ocular surfaces are fully separated by the tear layer, the mixed lubrication where a part of the eyelid and ocular surfaces is supported by the tear layer and in the other part, the eyelid surface may be in contact with the ocular surface, and the lubrication containing both the mixed and fluid lubrications.

\section{Acknowledgment}

This work was supported in part by the Menicon Co. Ltd.

\section{References}

[1] Lemp, M. A., Baudouin, C., Baum, J., Dogru, M., Foulks, G. N., \& Kinoshita, S., et al. (2007). The definition and classification of dry eye disease: Report of the definition and classification subcommittee of the international dry eye workshop (2007). Ocular Surface, 5(2), 75-92. 
[2] Korb, D. R., Herman, J. P., Greiner, J. V., Scaffidi, R. C., Finnemore, V. M., \& Exford, J. M., et al. (2005). Lid wiper epitheliopathy and dry eye symptoms. Eye \& Contact Lens, 31(1), 2-8.

[3] Berry, M., Pult, H., Purslow, C., \& Murphy, P. J. (2008). Mucins and ocular signs in symptomatic and asymptomatic contact lens wear. Optometry \& Vision Science, 85(10), E930-E938.

[4] Jones, M. B., Fulford, G. R., Please, C. P., McElwain, D. L. S., \& Collins, M. J. (2008). Elastohydrodynamics of the eyelid wiper. Bulletin of Mathematical Biology, 70(2), 323-343.

[5] Ehrmann, K., Francis, I., \& Stapleton, F. (2001). A novel instrument to quantify the tension of upper and lower eyelids. Contact Lens and Anterior Eye, 24(2), 65-72.

[6] Francis, I. C., Stapleton, F., Ehrmann, K., \& Coroneo, M. T. (2006). Lower eyelid tensometry in younger and older normal subjects. Eye, 20(2), 166-172.

[7] Vihlen, F. S., \& Wilson, G. (1983). The relation between eyelid tension, corneal toricity, and age. Investigative ophthalmology \& visual science, 24(10), 1367-1373.

[8] Sakai, E., Shiraishi, A., Yamaguchi, M., Ohta, K., \& Ohashi, Y. (2012). Blepharo-tensiometer: New eyelid pressure measurement system using tactile pressure sensor. Eye \& contact lens, 38(5), 326-330.

[9] Okamoto, S., Pranoto, S., Ohwaki, Y., Lee, J. H., Shiraishi, A., \& Sakane, Y., et al. (2016). Development of a physical apparatus and computational program employing a genetic algorithm and least-squares method for measuring the frictional coefficient of the human ocular surface. Proceedings of 3rd International Conference on Biomedical Engineering and Systems (ICBES'16). Budapest, Hungary.

[10] Salmon, S. (2011). Particle swarm optimization in scilab ver 0.1-7. Performance Evaluations.

[11] Pranoto, S., Okamoto, S., Lee, J. H., Shiraishi, A., Sakane, Y., \& Ohashi, Y. Determining frictional characteristics of human ocular surfaces by employing bsg-starcraft of particle swarm optimization. Journal of Biomedical Engineering and Biosciences.

[12] Hamrock, B. J., Schmid, S. R, \& Jacobson, B. O. (2004). Fundamentals of Fluid Film Lubrication. New York: Marker Dekker, Inc.

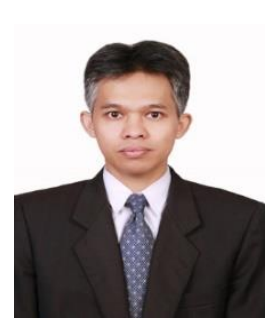

Sarwo Pranoto was born in Indonesia. He got BSc from Electrical Engineering Department, Gadjah Mada University, Indonesia in 2003. His master's degree was earned from Electrical and Computer System Engineering, Monash University, Australia in 2009. He is currently studying in doctoral course of Mechanical Engineering, Graduate School of Science and Engineering, Ehime University.

In 2005, he joined the Department of Electrical Engineering, State Polytechnic of Ujung Pandang, Indonesia as a lecturer. His research interests include biomedical engineering and wireless sensor networks in health applications.

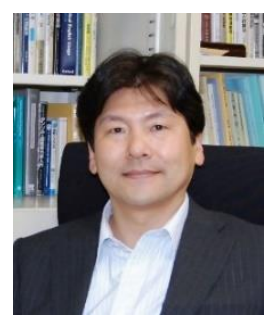

Shingo Okamoto received the bachelor's degree in engineering in 1982 and the master's degree in engineering in 1984 from Ehime University. He also obtained the doctor's degree in engineering in 1992 from Tokyo Institute of Technology.

He had been doing researches as a researcher in Research and Development Center of Toshiba Corp. from 1984, as a research associate from 1992 and an associate professor from 1994 in Hiroshima University. During the time, from 1998 to 1999, he had been doing a research as visiting scientist with Prof. K. J. Bathe in Massachusetts Institute of Technology. Then he has been a professor of Mechanical Engineering Course of Ehime University from 2008 to the present time. He is interested in robotics, intelligent systems, artificial intelligence, biomedical engineering science and computational mechanics. 
In the field of biomedical engineering science, he gained a Best Paper Award in the international conference on ECBA (Engineering \& Technology, Computer, Basic \& Applied Science) and a Technology Prize from the SICE (Society of Instrument and Control Engineers) in 2016.

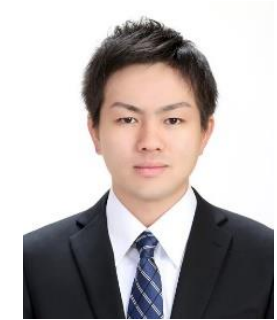

surfaces.

Ryoichiro Kataoka was born in Japan. He got the bachelor's degree from the Department of Mechanical Engineering, Faculty of Engineering, Ehime university, Japan in 2016. He had conducted a graduation research in the field of robotics.

He is currently studying in master course of mechanical engineering, Graduate School of Science and Engineering, Ehime University. He participated in development of the ocular surface tribometer and analysis of the frictional characteristics of human ocular

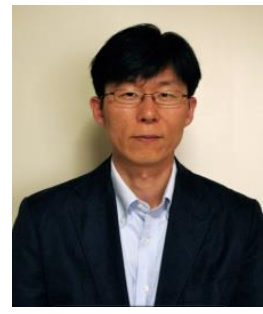

Jae Hoon Lee received the B.S. degree in mechanical and automatic control engineering from Korea University of Technology and Education, in 1996, and the M.S. and Ph.D. degrees in electric, electrical, control and instrumentation engineering at Hanyang University, Korea, in 1998 and 2003, respectively. From 2004 to 2006, he was a postdoctoral fellow at the Intelligent Robot Laboratory, University of Tsukuba, Japan. From 2007 to 2008, he was a JSPS fellow with the Ubiquitous Functions Research Group, Intelligent Systems Research Institute, AIST, Japan.

He is currently an associate professor in the Department of Mechanical Engineering, Graduate School of Science and Engineering, Ehime University, Japan. His current research interests include the collaborative navigation of mobile robot in daily environment, the development of drone system for inspection tasks, and the design and control of bio-inspired robotic systems.

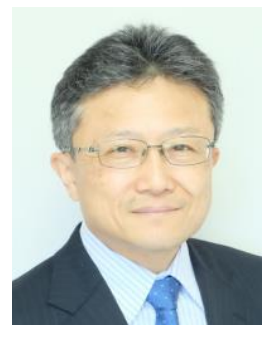

Atsushi Shiraishi received the M.D. from Nippon Medical School in 1986. He got the Ph.D. from Graduate School of Medicine, Nippon Medical School, Japan in 1995. His research interests are in the field of ophthalmology.

Currently, he is a professor and the chair of the Department of Ophthalmology, Ehime University Graduate School of Medicine.

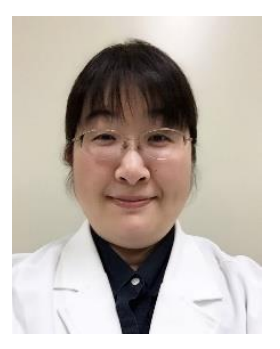

Yuri Sakane received M.D. from Ehime University School of Medicine in 2004. Currently, she joins the Department of Ophthalmology Ehime University Graduate School of Medicine. Her research interests are in the field of ophthalmology.

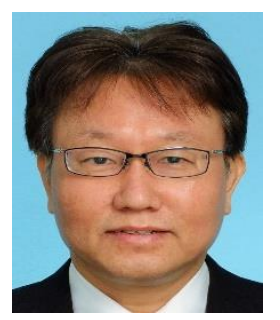

Masahiko Yamaguchi received M.D. from College of Ophthalmology, Osaka City University, Osaka, Japan in 1990. He was an assistant professor of Ophthalmology, Osaka City University, Osaka, Japan in 1993. Then in 1996, he became an assistant professor ophthalmology, Ehime University School of Medicine, Ehime, Japan.

In 2001, he became a vice director of Ophthalmology, Matsuyama Red Cross Hospital, Ehime, Japan. He joined as a lecturer of ophthalmology at Ehime University School of 
Medicine, Ehime, Japan in 2008. Then, in 2011, he joined as a lecturer of Ophthalmic Refractive Center, Ehime University Hospital, Ehime, Japan. In 2013, he became associate professor of Regional Ophthalmology, Ehime University School of Medicine, Ehime, Japan. Since 2015, he has been the director of Ophthalmology, Ehime Prefectural Central Hospital, Ehime, Japan.

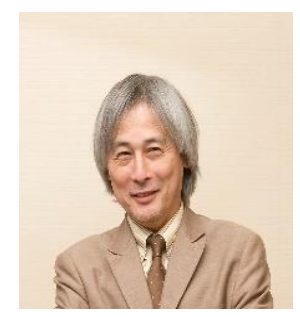

Yuichi Ohashi received M.D. from Osaka University Medical School, in 1975. In 1978, he became a research associate in the Department of Pathology, Research Institute for Microbial Diseases, Osaka University. In 1980, he was a clinical instructor in the Department of Ophthalmology, Osaka University Medical School. In 1982, he was a senior research associate, Francis I, Proctor Foundation, in University of California San Francisco. His research interests are in the field of ophthalmology.

In 1985, he became the Chief of Division of Ophthalmology, Kansai Rosai Hospital. Then in 1989, he became an Assistant Professor in Department of Ophthalmology, Osaka University Medical School. In 1992, he has been a Professor of Department of Ophthalmology, Ehime University School of Medicine and at that time he became the chairman of Department of Ophthalmology, Ehime University School of Medicine. In 2003 and 2006 he became the Director of Ehime University Hospital and an Executive Director of Ehime University, respectively. Then he became a Vice President of Ehime University in 2009. He has been the president of Ehime University from 2015 to the present time. 